\title{
Evisceration of Small Bowel through Femoral Triangle Following Exanguinating Bull Gore Injury
}

\author{
CA Shashirekha, K Krishnaprasad, Vijay Agarwal
}

\begin{abstract}
Bull gore injuries are a common feature among farmers and revellers in rural India as well as in the West, such as Pamplona, Spain. ${ }^{1}$ All types of bull gore injuries of varying severity, both penetrating and blunt types, sometimes causing death are described. One such successfully managed case of bull gore injury of abdomen through right femoral triangle, surprisingly sparing the femoral vessels but causing exanguinating contusion and laceration of mesorectum and mesentery and evisceration of small bowel through femoral triangle is being reported to highlight the varied presentation of bull gore injuries, our rural folk face day in and day out. Successful outcome is inevitable following prompt and learned surgical intervention.
\end{abstract}

Keywords: Bull gore, Penetrating/blunt injury, Femoral triangle, Evisceration.

How to cite this article: Shashirekha CA, Krishnaprasad K, Agarwal V. Evisceration of Small Bowel through Femoral Triangle Following Exanguinating Bull Gore Injury. Euroasian J Hepato-Gastroenterol 2012;2(1):54-55.

\section{Source of support: Nil}

Conflict of interest: None declared

\section{INTRODUCTION}

A 70-year-old male was brought to casualty after being gored by a bull. He was hemodynamically unstable and arrived complaining of severe right lower quadrant abdominal pain. He had no injuries to the head and chest other than minor abrasions on left upper limb.

Initial assessment showed pulse of 86/minutes, blood pressure of $50 \mathrm{~mm} \mathrm{Hg}$ systolic.

There was $7 \times 5 \mathrm{~cm}$ penetrating wound $2 \mathrm{~cm}$ below the right inguinal region. Herniation of about $35 \mathrm{~cm}$ of small bowel showing visible peristalsis found. There was active bleed from the wound (Fig. 1).

The patient was taken to operation room for emergency exploratory laparotomy. The abdomen was explored through a midline incision. Iliopsoas muscle was contused and lacerated and there were multiple contusions and bleeding laceration of mesentery, mesocolon and mesorectum, which were managed by pressure and ligation (Figs $2 \mathrm{~A}$ to D).

Bowel was normal. Femoral vessels was found intact.

Herniated bowel thoroughly cleaned, reduced and rent in the femoral triangle repaired.

Postoperatively the patient's condition was gradually improved.

Pelvic drain was removed on postoperative day 5 and sutures were removed on day 8 .

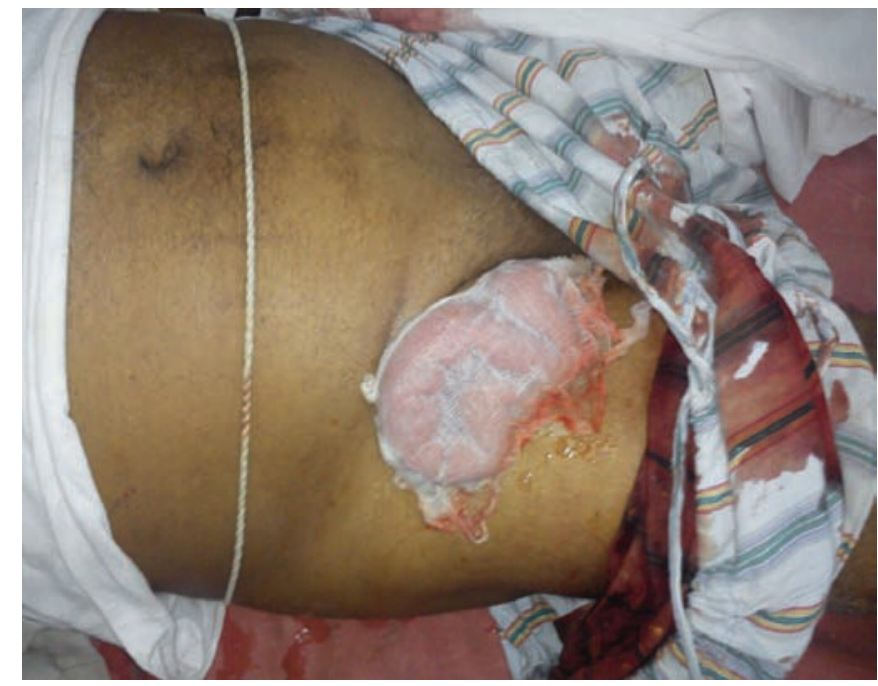

Fig. 1: Site of penetrating injury with traumatic hernia

The patient's recovery was uneventful and was discharged on the 12th postoperative day with regular follow-up of no complication.

\section{DISCUSSION}

In India, bull gore injuries are more commonly seen in rural areas.

Dogan et $\mathrm{al}^{2}$ in a study of persons with occupations in animal husbandry concluded that bulls were among the most dangerous of all the animals.

Bull horn impact may vary from contusions, laceration and penetrating wounds involving internal organs to fractures.

The injuries occur more commonly on the abdomen and perineum. ${ }^{3,4}$

In this case, the injury occurred predominantly on right lower side of abdomen. The explanation for this could be sufferer was just about to run away from the attacking animal and thus turning right region of the body toward the animal.

\section{CONCLUSION}

Bull gore injuries are a challenge for rural surgeons since they may be trivial or fatal.

They may be grotesque appearing and need not be fatal since integrity of great vessels cannot be breached easily.

Appropriate visceral injury repair combined with thorough wound debridement and broad spectrum antibiotics ${ }^{5}$ will lead to certain recovery. 

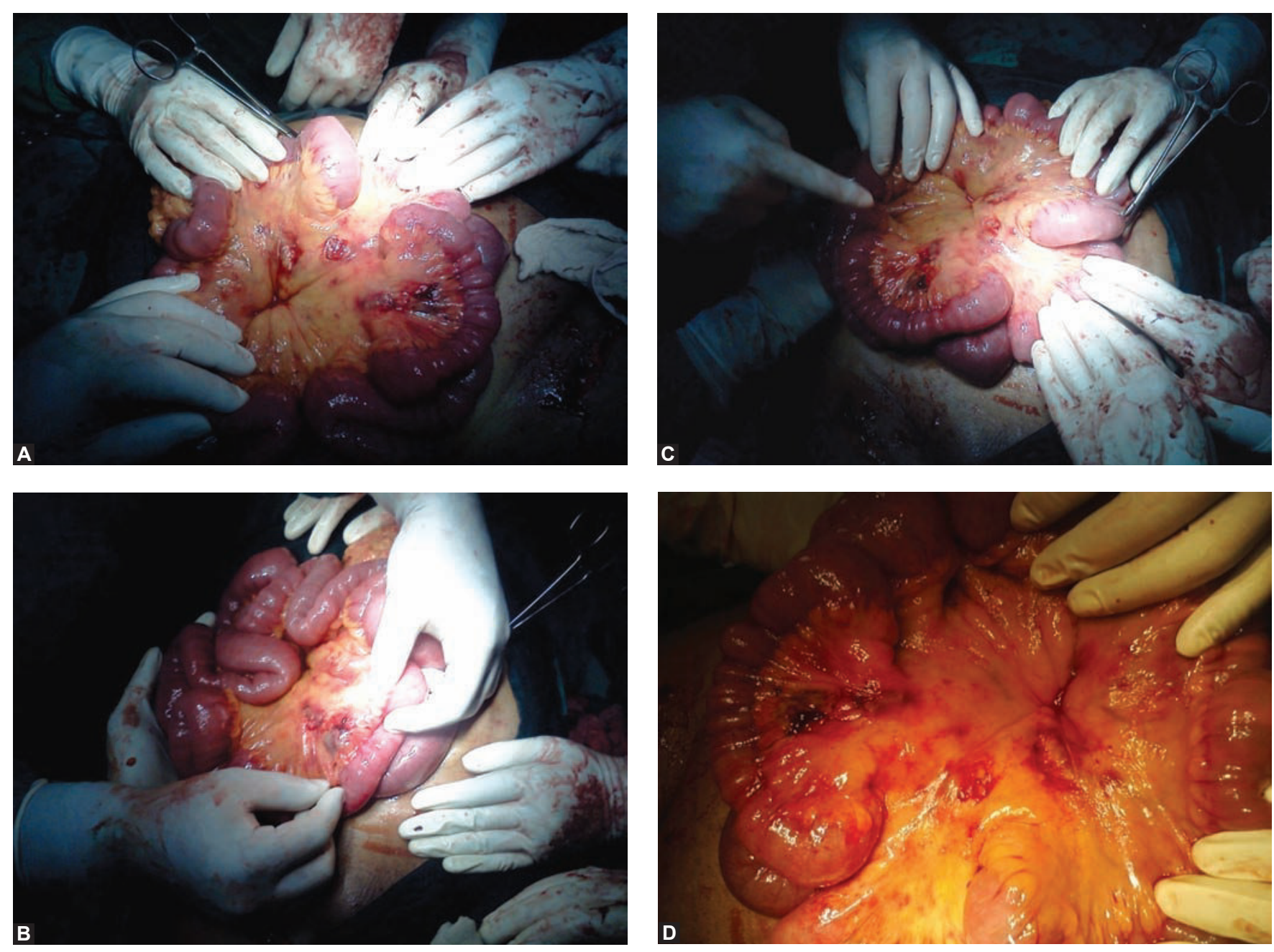

Figs 2A to D: Multiple mesentery, mesocolic, mesorectal tears and contusions

\section{REFERENCES}

1. Shukla HS, Mittal DK, Naithani YP. Bull horn injury-a clinical study. The British Journal of Accident Surgery 1977;9:164-67.

2. Dogan KH, Demirci S, Erkol Z, Sunam GS, Kucukkartallar TJ. Injuries and deaths occurring as a result of bull attack. Agromedicine 2008;13:191-96.

3. Sekhon MS, Khatri HL, Grewal SS, Marya SKS. Bull horn injury. Indian J Surgery 1983;45:486-88.

4. Rau JBV. Bull gore injuries in rural areas. Indian J Surgery 1982;44:664-71.

5. Rani M, Rohit, Sharma A, Dikshit PC. Injuries by bull horns: Patterns and prevention protocols. Anil Aggrawal's Internet Journal of Forensic Medicine and Toxicology. January-June 2010;11(1) [serial online]. Available at: http:// www.anilaggrawal.com/ij/vol_011_no_001/main.html. Accessed October 19, 2011.

\section{ABOUT THE AUTHORS}

\section{CA Shashirekha (Corresponding Author)}

Department of General Surgery, Sri Devaraj Urs Medical College Kolar, Karnataka-563101, India, Phone: +91-9986510936, e-mail: drca_shashirekha@yahoo.co.in

\section{K Krishnaprasad}

Department of General Surgery, Sri Devaraj Urs Medical College Kolar, Karnataka, India

\section{Vijay Agarwal}

Department of General Surgery, Sri Devaraj Urs Medical College Kolar, Karnataka, India 